\title{
The $\mu$ Subunit of Arabidopsis Adaptor Protein-2 Is Involved in Effector-Triggered Immunity Mediated by Membrane-Localized Resistance Proteins
}

\author{
Noriyuki Hatsugai, ${ }^{1}$ Rachel Hillmer, ${ }^{1}$ Shohei Yamaoka, ${ }^{2}$ Ikuko Hara-Nishimura, ${ }^{2}$ and Fumiaki Katagiri1 \\ ${ }^{1}$ Department of Plant Biology, Microbial and Plant Genomics Institute, University of Minnesota, 1500 Gortner Ave., St. Paul, \\ MN 55108, U.S.A.; and ${ }^{2}$ Department of Botany, Graduate School of Science, Kyoto University, Kitashirakawa, Sakyo-ku, \\ Kyoto 606-8502, Japan
}

Submitted 9 October 2015. Accepted 25 January 2016.

\begin{abstract}
Endocytosis has been suggested to be important in the cellular processes of plant immune responses. However, our understanding of its role during effector-triggered immunity (ETI) is still limited. We have previously shown that plant endocytosis, especially clathrin-coated vesicle formation at the plasma membrane, is mediated by the adaptor protein-2 (AP-2) complex and that loss of the $\mu$ subunit of AP-2 (AP2M) affects plant growth and floral organ development. Here, we report that AP2M is required for full-strength ETI mediated by the disease resistance $(R)$ genes $R P M 1$ and $R P S 2$ in Arabidopsis. Reduced ETI was observed in an ap $2 m$ mutant plant, measured by growth of Pseudomonas syringae pv. tomato DC3000 strains carrying the corresponding effector genes avrRpm1 or avrRpt 2 and by hypersensitive cell death response and defense gene expression triggered by these strains. In contrast, RPS4-mediated ETI and its associated immune responses were not affected by the ap2m mutation. While RPM1 and RPS2 are localized to the plasma membrane, RPS4 is localized to the cytoplasm and nucleus. Our results suggest that AP2M is involved in ETI mediated by plasma membrane-localized $R$ proteins, possibly by mediating endocytosis of the immune receptor complex components from the plasma membrane.
\end{abstract}

Plant defenses that restrict pathogen growth are often mediated by resistance $(\mathrm{R})$ proteins. The most prevalent class of $\mathrm{R}$ proteins is the nucleotide-binding leucine-rich repeat (NB-LRR) type (Dangl and Jones 2001; Tameling and Joosten 2007). Other classes are the receptor-like kinase (RLK) and receptor-like protein (RLP) types, both of which possess an extracellular LRR domain and a transmembrane domain. While members of the RLK class contain a cytoplasmic serine and threonine kinase domain, the members of the RLP class do not contain a recognizable cytoplasmic signaling domain (Dangl and Jones 2001; Tameling and Joosten 2007). These R proteins

Current address for S. Yamaoka: Graduate School of Biostudies, Kyoto University, Kyoto, Japan.

Corresponding authors: F. Katagiri; E-mail: katagiri@umn.edu and I. HaraNishimura; E-mail: ihnishi@gr.bot.kyoto-u.ac.jp

*The $\boldsymbol{e}$-Xtra logo stands for "electronic extra" and indicates that a supplementary table is published online.

This article is in the public domain and not copyrightable. It may be freely reprinted with customary crediting of the source. The American Phytopathological Society, 2016. recognize pathogen-specific signals called effectors and this recognition leads to induction of effector-triggered immunity (ETI). ETI is often accompanied by a hypersensitive response (HR), which is characterized by rapid and localized programmed cell death (hypersensitive cell death) (Hara-Nishimura and Hatsugai 2011; Jones and Dangl 2006; Tsuda and Katagiri 2010).

In Arabidopsis, two NB-LRR R proteins, RPM1 and RPS2, interact with RIN4 at the plasma membrane (Axtell and Staskawicz 2003; Mackey et al. 2003). RPM1 specifically detects phosphorylation of RIN4 triggered by the Pseudomonas syringae effector AvrRpm1 and leads to RPM1-mediated ETI, whereas RPS2 recognizes proteolytic cleavage of RIN4 by the AvrRpt2 effector and leads to RPS2-mediated ETI (Axtell and Staskawicz 2003; Mackey et al. 2003). Another NB-LRR R protein, RPS4, confers resistance to $P$. syringae expressing AvrRps4. RPS4 distributes between the cytoplasm and nucleus, and nuclear localization of RPS4 is required for RPS4-mediated ETI (Wirthmueller et al. 2007).

Clathrin-mediated endocytosis (CME) is the major endocytosis pathway in animal cells. During CME, cargo proteins are captured predominantly by the adaptor protein-2 (AP-2) complex and are assembled into transport vesicles coated with clathrin. AP-2 is a heterotetrameric complex consisting two large subunits ( $\alpha$ and $\beta 2$ ), a medium subunit $(\mu 2)$, and a small subunit $(\sigma 2)$. For the capture of cargo proteins, $\mu 2$ recognizes the tyrosinebased YXXФ motif ( $\Phi$ represents Leu, Ile, Phe, Met, or Val) present in the cytosolic domains of cargo proteins (Ohno et al. 1995). Multiple lines of evidence have shown that plants also use CME for uptake of various proteins from the plasma membrane, including the PIN-FORMED (PIN) auxin transporters, the water channel PLASMA MEMBRANE INTRINSIC PROTEIN2 (Dhonukshe et al. 2007), the boron transporter REQUIRES HIGH BORON1 (Takano et al. 2010), the iron transporter IRON-REGULATED TRANSPORTER1 (Barberon et al. 2011), the plant-specific endocytic SNARE VESICLE-ASSOCIATED MEMBRANE PROTEIN727 (Ebine et al. 2011), and the ligandactivated brassinosteroid receptor BRASSINOSTEROID INSENSITIVE1 (Irani et al. 2012). We and other groups have recently shown that AP-2 is involved in plant CME (Di Rubbo et al. 2013; Fan et al. 2013; Kim et al. 2013; Yamaoka et al. 2013). An insertional mutation in the $A P 2 M$ gene encoding the Arabidopsis $\mu 2$ subunit causes reduced fertility and abnormal floral organ development (Kim et al. 2013; Yamaoka et al. 2013).

AP-2 has been suggested to be involved in R protein-mediated immunity (reviewed by Geldner and Robatzek 2008; LeborgneCastel et al. 2010; Bar and Avni 2014; Teh and Hofius 2014). 
A
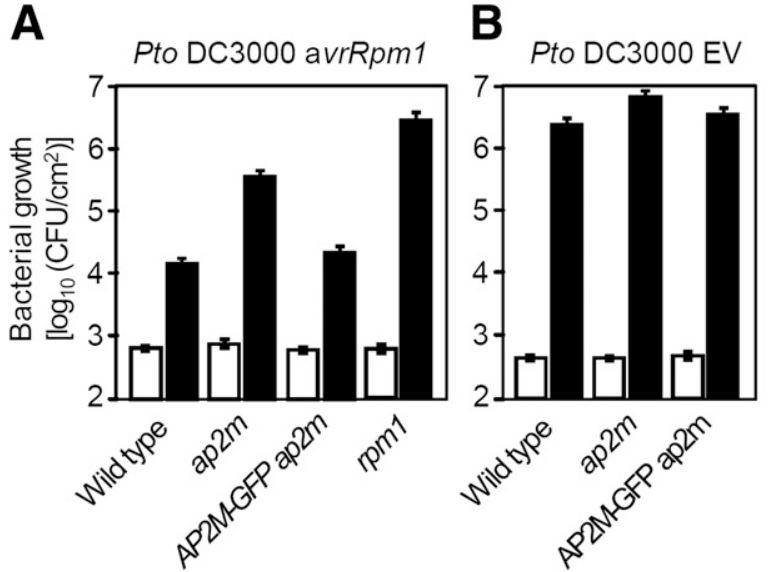

C

\begin{tabular}{|c|c|c|}
\hline Genotype & \multicolumn{1}{c}{ ETI } & \multicolumn{1}{c|}{$P$-value } \\
\hline Col-0 & 2.13 & $\ldots \ldots \ldots$ \\
\hline ap2m & 1.21 & $8.4 \times 10^{-7}$ \\
\hline $\begin{array}{c}A P 2 M- \\
G F P a p 2 m\end{array}$ & 2.09 & 0.92 \\
\hline
\end{tabular}

ETI: $\log _{10}\left(\mathrm{CFU} / \mathrm{cm}^{2}\right)$

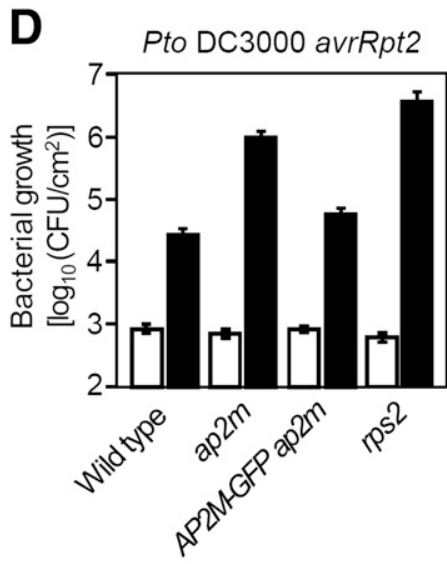

$\mathbf{F}$

\begin{tabular}{|c|c|c|}
\hline \multicolumn{1}{c}{ Genotype } & \multicolumn{1}{c}{ ETI } & \multicolumn{1}{c|}{$P$-value } \\
\hline Col-0 & 1.90 & $\ldots \ldots \ldots$ \\
\hline$a p 2 m$ & 0.76 & $1.5 \times 10^{-9}$ \\
\hline $\begin{array}{c}A P 2 M- \\
G F P a p 2 m\end{array}$ & 1.76 & 0.37 \\
\hline
\end{tabular}

ETI: $\log _{10}\left(\mathrm{CFU} / \mathrm{cm}^{2}\right)$

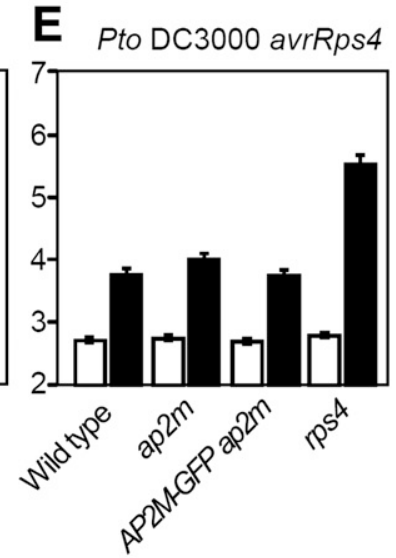

G

\begin{tabular}{|c|c|c|}
\hline Genotype & ETI & $P$-value \\
\hline Col-0 & 2.34 & $\ldots \ldots \ldots$ \\
\hline$a p 2 m$ & 2.49 & 0.82 \\
\hline $\begin{array}{c}A P 2 M-G F P \\
\text { ap2m }\end{array}$ & 2.51 & 0.44 \\
\hline \multicolumn{3}{|c|}{ ETI: $\log _{10}\left(\mathrm{CFU} / \mathrm{cm}^{2}\right)$}
\end{tabular}

Fig. 1. AP2M deficiency compromised resistance to Pseudomonas syringae pv. tomato DC3000 avrRpm1 and DC3000 avrRpt2 but not to DC3000 avrRps4. A, P. syringae pv. tomato DC3000 avrRpm1, B, DC3000 EV (empty vector), D, DC3000 avrRpt2, and E, DC3000 avrRps4 (optical density at $600 \mathrm{~nm}=0.0001$ ) were inoculated into leaves of wild type, ap $2 m$, AP2M-GFP ap2m, and the corresponding resistance gene mutants. Live bacteria were counted at 0 days postinoculation (dpi) (open bar) and $2 \mathrm{dpi}$ (filled bar). Bars represent the mean and error bars the standard error from model fitting. The data were collected in three independent experiments, each of which had six or eight biological replicates. C, F, and G, The level of effector-triggered immunity (ETI) was estimated by subtracting $\log _{10}$-transformed bacterial counts at $2 \mathrm{dpi}$ for plants inoculated with P. syringae pv. tomato DC3000 avrRpm1, DC3000 avrRpt2, or DC3000 avrRps 4 , respectively, from the $\log _{10}$-transformed counts for $P$. syringae $\mathrm{pv}$. tomato DC3000 EV. The ETI level of each plant was compared with that of wild type, using a two-tailed $t$ test to obtain the $P$ values (Holm-Bonferroni corrected).

For example, the YXXФ motif is present in the cytoplasmic domain of the tomato (Lycopersicon esculentum) RLP receptor LeEix2 (Ron and Avni 2004). Mutation of this motif in LeEix2 blocked xylanase-induced endocytosis and abolished its ability to induce HR, suggesting that the AP-2 complex might be involved in xylanase-triggered HR (Ron and Avni 2004; Sharfman et al. 2011). The YXXФ motif is found in the cytoplasmic domain of other RLPs, such as Ve2, Cf4, and Cf9 (Jones et al. 1994; Kawchuk et al. 2001; Takken et al. 1998), but the functional relevance of the motif for their $\mathrm{R}$ protein-mediated immunity is still to be unraveled. Another example of the involvement of endocytosis in $\mathrm{R}$ protein-mediated immunity has been proposed in rice (Oryza sativa). During RLK Xa21mediated race-specific resistance to the bacterial blight agent Xanthomonas oryzae pv. oryzae (Chen et al. 2010), Xa21 was internalized and probably transported via the trans-Golgi network and early endosome compartment. Additionally, cryptogein, an elicitor from the oomycete Phytophthora cryptogea, stimulated CME in tobacco BY-2 cells (Leborgne-Castel et al. 2008). Similarly, during infection by the hemibiotrophic fungus Magnaporthe oryzae, rice cells exhibited increased endocytosis (Kankanala et al. 2007). However, no direct evidence has been provided for the involvement of AP-2 in plant immune responses.

In this study, we investigated immune responses mediated by three different NB-LRR R proteins, i.e., RPM1, RPS2, and RPS4, and found that $A P 2 M$ deficiency reduces ETI against P. syringae pv. tomato DC3000 avrRpm1 and DC3000 avrRpt2 but not against DC3000 avrRps4. Our results provide direct evidence of the role of CME in plant immunity.

\section{RESULTS}

An ap2m mutant showed reduced levels of ETI against $P$. syringae pv. tomato DC3000 avrRpm1 and DC3000 avrRpt2 but not to DC3000 avrRps4.

The Col- 0 accession of Arabidopsis carries an $R$ gene, $R P M 1$, which confers resistance to $P$. syringae pv. tomato DC3000 avrRpml (Grant et al. 1995). To investigate whether AP-2 is involved in RPM1-mediated ETI, we examined whether resistance to $P$. syringae pv. tomato DC3000 avrRpml was impaired in an ap2m T-DNA insertion mutant (SAIL_165_A05) (Yamaoka et al. 2013). We inoculated wild-type and ap $2 m$ plants with $P$. syringae pv. tomato DC3000 avrRpml and counted viable bacteria immediately and 2 days postinoculation (dpi). The number of bacteria in ap $2 m$ plants was higher than that in wild-type plants at 2 dpi but lower than the number in rpml plants used as a control (Fig. 1A). Bacterial growth of the virulent strain $P$. syringae pv. tomato DC3000 carrying an empty vector ( $P$. syringae pv. tomato DC3000 EV) in ap $2 m$ plants was only slightly increased compared with wild-type plants (Fig. 1B). The difference in $\log _{10}$-transformed numbers of bacteria between $P$. syringae pv. tomato DC3000 avrRpml and DC3000 EV at 2 dpi was used to estimate the impact of the ap $2 m$ mutation on RPM1-mediated ETI. We observed a $43 \%$ reduction of RPM1-mediated ETI in the ap $2 m$ plants compared with wild-type plants $\left(P=8.4 \times 10^{-7}\right)$ (Fig. 1C). An ap $2 m$ complementation line, which carries an $A P 2 M-G F P$ (green fluorescent protein) transgene under the control of the endogenous $A P 2 M$ promoter ( $A P 2 M-G F P$ ap2m) (Yamaoka et al. 2013), gave results similar to wild-type plants, confirming that the results obtained with the ap $2 m$ mutant were indeed the effect of the ap $2 m$ mutation. Thus, an $A P 2 M$ deficiency results in compromised RPM1-mediated ETI.

To examine the effect of $A P 2 M$ deficiency on ETI mediated by another R protein, RPS2, wild-type, ap $2 m, A P 2 M-G F P$ ap $2 m$, and rps 2 plants were inoculated with $P$. syringae pv. tomato DC3000 avrRpt2 (Fig. 1D). RPS2-mediated ETI was reduced 
by $60 \%$ in ap $2 m$ plants compared with ETI in wild-type plants $\left(P=1.5 \times 10^{-9}\right)($ Fig. $1 \mathrm{~F})$; this effect was complemented in $A P 2 M-G F P$ ap $2 m$ plants. These results indicate that AP2M is involved in RPS2-mediated ETI as well as RPM1mediated ETI.

AP2M plays a central role in CME from the plasma membrane (Di Rubbo et al. 2013) (Fan et al. 2013; Kim et al. 2013; Yamaoka et al. 2013). In contrast to plasma membrane-associated RPM1 and RPS2, RPS4 is localized to the nucleus and cytoplasm (Wirthmueller et al. 2007). It is unlikely that RPS4-mediated ETI is affected by $A P 2 M$ deficiency. To test this hypothesis, we measured growth of $P$. syringae pv. tomato DC3000 avrRps 4 in wild type, ap $2 m, A P 2 M-G F P$ ap $2 m$, and rps 4 plants (Fig. 1E). No significant difference in RPS4-mediated ETI was observed among wild type, ap $2 m$, and AP2M-GFP ap $2 m$ (Fig. 1G).

Taken together, we conclude that AP2M is involved in both RPS2-mediated and RPM1-mediated ETI but not involved in RPS4-mediated ETI.

\section{A}

A Pto DC3000 avrRpm1
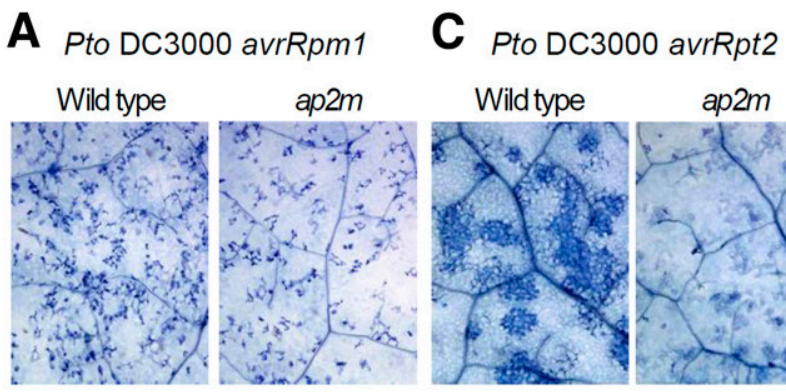

AP2M-GFP ap2m $\quad$ pm1 $p s 2$
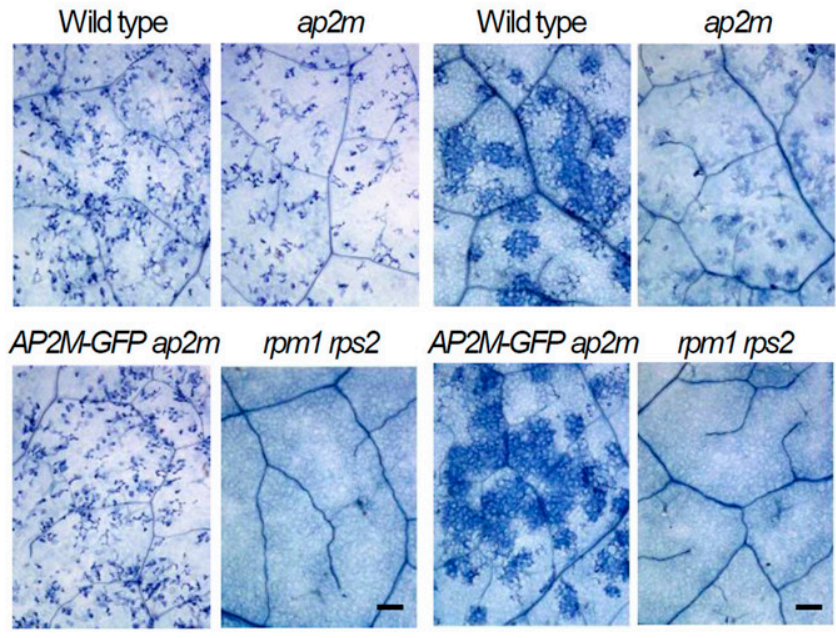

\section{B}

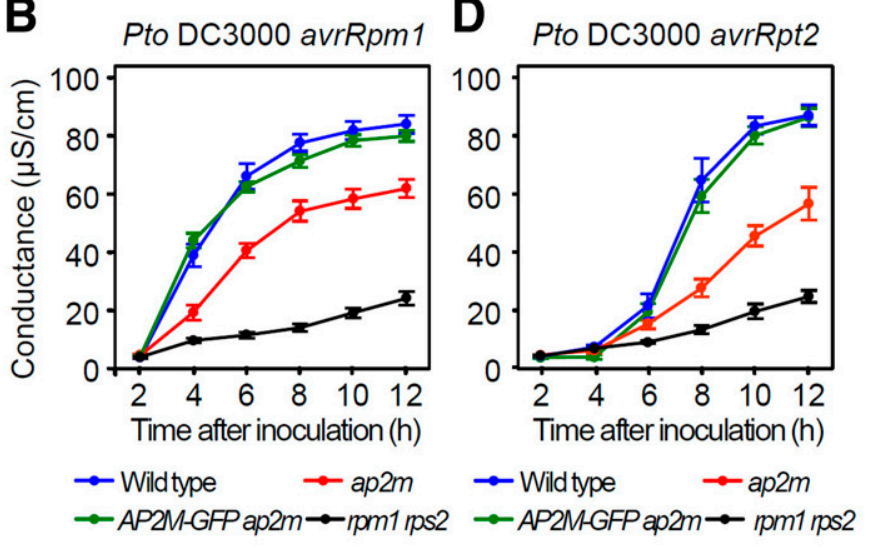

The $A P 2 M$ deficiency reduced the amount of hypersensitive cell death during RPM1- and RPS2-mediated ETI.

ETI is often associated with hypersensitive cell death (Dangl and Jones 2001; Jones and Dangl 2006). To determine whether $A P 2 M$ deficiency affects hypersensitive cell death, we monitored cell death in wild-type and $a p 2 m$ plants, using trypan blue staining $12 \mathrm{~h}$ postinoculation with $P$. syringae pv. tomato DC3000 avrRpm1. Dead cells are seen as a distinctive blue color under a light microscope in wild-type plants (Fig. 2A). The ap $2 m$ mutant had clearly fewer dead cells than wild type (Fig. 2A). The number of dead cells in AP2M-GFP ap $2 m$ was similar to that observed in wild type, while no dead cells were observed in rpm 1 rps 2 . We obtained consistent results when the cell death was quantified using an electrolyte leakage assay (Fig. 2B); the extent of cell death was high and comparable between wild type and $A P 2 M-G F P$ ap $2 m$, while it was clearly lower in $a p 2 m$, and a very low level of cell death was observed with rpm1 rps2.
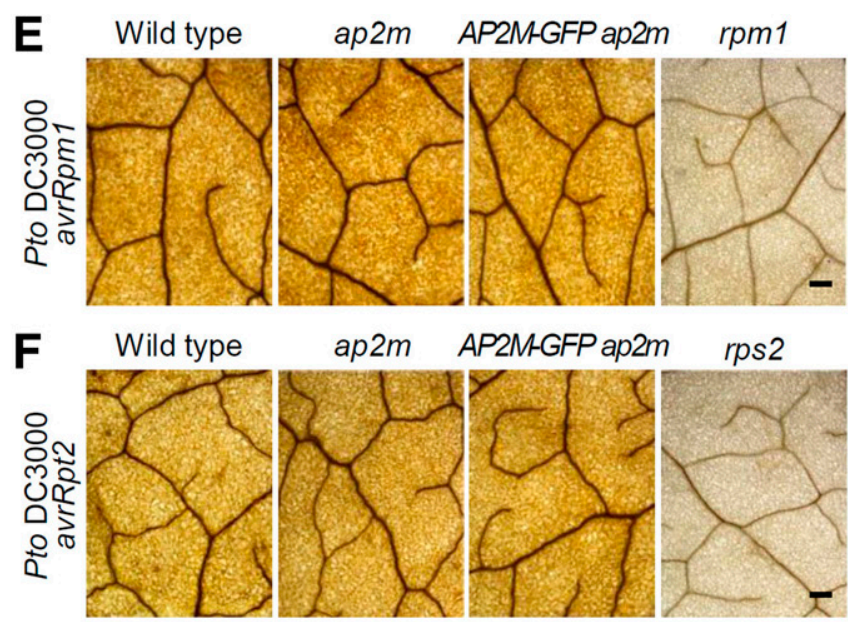

AP2M-GFP ap2m

rps2
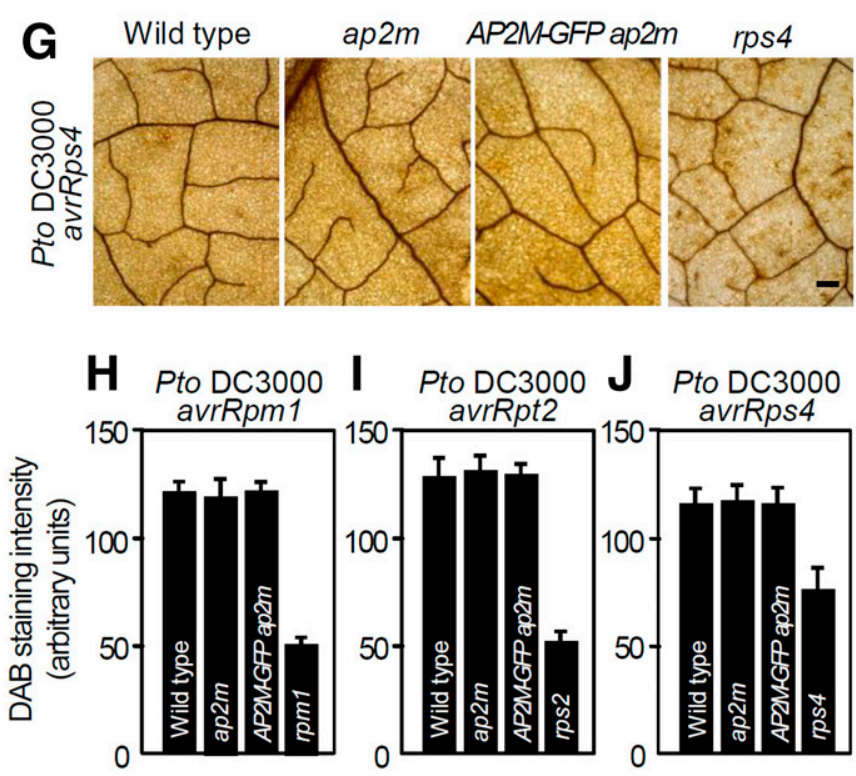

Fig. 2. Hypersensitive cell death was reduced in the ap $2 m$ mutant, although reactive oxygen species production was not impaired. A, Pseudomonas syringae pv. tomato DC3000 avrRpml and C, DC3000 avrRpt2 (optical density at $600 \mathrm{~nm}\left[\mathrm{OD}_{600}\right]=0.001$ ) were inoculated into leaves of wild type, ap2m, AP2M-GFP ap2m, and rpm1 rps 2 . Dead cells in the leaves at $12 \mathrm{~h}$ after the inoculation were visualized by trypan-blue staining. Bar $=200 \mu \mathrm{m}$. B, P. syringae pv. tomato DC3000 avrRpm1 and D, DC3000 avrRpt $2\left(\mathrm{OD}_{600}=0.1\right)$ were infiltrated into leaves of wild type, ap $2 m, A P 2 M-G F P$ ap $2 m$, and $r p m 1 ~ r p s 2$. Electrolyte leakage from dying and dead cells in the leaves at 2 to $12 \mathrm{~h}$ after the inoculation was measured. Vertical bars indicate standard errors of three independent experiments, each of which had four biological replicates. E, . syringae pv. tomato DC3000 avrRpm1, F, DC3000 avrRpt2, and G, DC3000 avrRps4 $\left(\mathrm{OD}_{600}=0.1\right)$ were inoculated into leaves of wild type, ap2m, AP2M-GFP ap $2 \mathrm{~m}$, and the corresponding resistance $(R)$ gene mutants. The leaves were stained with $3,3^{\prime}$-diaminobenzidine (DAB) $12 \mathrm{~h}$ (for $P$. syringae pv. tomato DC3000 avrRpml) and $24 \mathrm{~h}$ (for P. syringae pv. tomato DC3000 avrRpt2 and DC3000 avrRps4) after the inoculation. Bar = $200 \mu \mathrm{m}$. H, I, and $\mathbf{J}, \mathbf{Q u a n t i f i c a t i o n}$ of the $\mathrm{DAB}$ staining intensity. Bars represent the mean and error bars the standard deviation from five stained regions in three different leaves. 
Similar results were obtained for hypersensitive cell death during RPS2-mediated ETI by both trypan blue staining (Fig. 2C) and electrolyte leakage (Fig. 2D). In both analyses, the extent of cell death was high and comparable between wild type and AP2M-GFP ap2m, and the level of cell death was clearly lower in ap2m. No or a very low level of cell death was observed with rpml rps2. Thus, hypersensitive cell death during ETI mediated by RPM1 and RPS2 is affected by the ap $2 m$ mutation.

The RPS4-mediated hypersensitive cell death is extremely weak in wild-type Col-0 plants (Gassmann et al. 1999; Tornero et al. 2002), which made it impossible to assess the effect

A

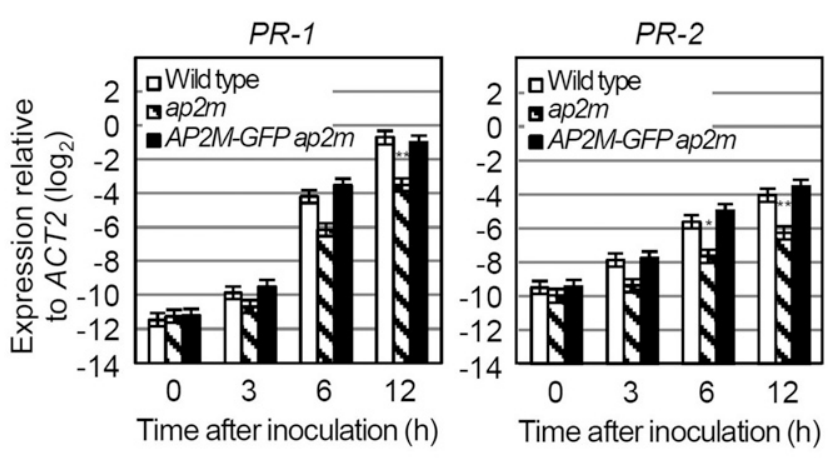

B

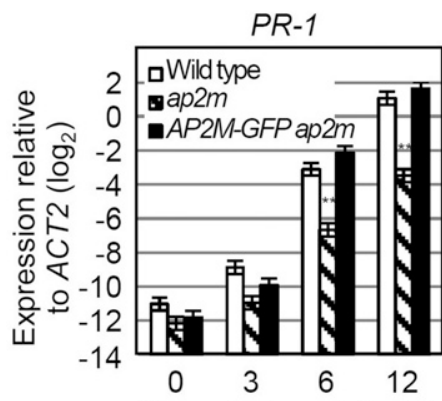

Time after inoculation ( $\mathrm{h}$ )

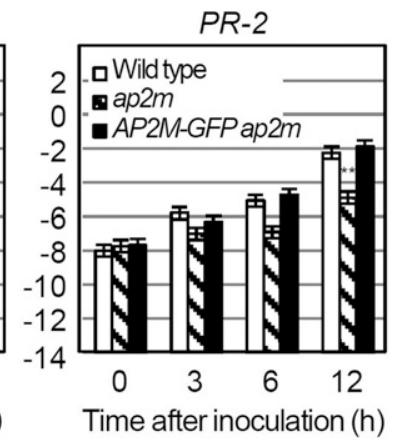

C

Pto DC3000 avrRps4
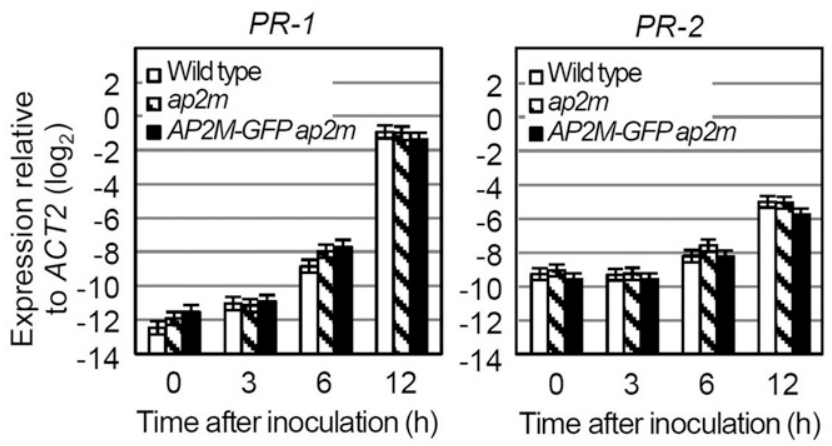

Fig. 3. Induced mRNA levels of two pathogenesis-related $(P R)$ genes in ap $2 \mathrm{~m}$ leaves were lower than those in wild-type leaves. A, Pseudomonas syringae pv. tomato DC3000 avrRpm1, B, DC3000 avrRpt2, and C, DC3000 avrRps4 (optical density at $600 \mathrm{~nm}=0.1$ ) were inoculated into leaves of wild type, ap $2 m$, and $A P 2 M-G F P$ ap $2 m$ and tissue was harvested immediately $(0 \mathrm{~h})$ and 3,6 , and $12 \mathrm{~h}$ after the inoculation. Each bar represents the $\log _{2}$ of the mean expression value and the error bars represent the standard error from fitting a model to the data collected in three independent biological replicates. The $\log _{2}$-transformed expression values were normalized using the control gene ACTIN2 (At2g18780). Asterisks indicate significant differences compared with wild-type plants (* indicates $P<0.05$, ** indicate $P<0.01$, twotailed $t$ tests adjusted with the Holm-Bonferroni correction). of $A P 2 M$ deficiency on hypersensitive cell death during RPS4-mediated ETI.

ROS production during ETI was normal in ap $2 m$ mutant.

It has been proposed that ROS production is involved in hypersensitive cell death (Torres et al. 2006). To examine the involvement of AP2M in ROS production during RPM1-mediated immune responses, we inoculated leaves with $P$. syringae pv. tomato DC3000 avrRpml and, then, stained them with 3,3'diaminobenzidine (DAB), which reacts with $\mathrm{H}_{2} \mathrm{O}_{2}$ to produce brown polymerized precipitate. The brown-colored precipitate accumulated to equivalent levels in leaves of wild-type, ap $2 m$, and AP2M-GFP ap $2 m$ plants (Fig. $2 \mathrm{E}$ and $\mathrm{H}$ ). These results suggest that AP2M is not required for ROS production during RPM1-mediated immune responses. Similarly, deficiency of $A P 2 M$ had no effect on ROS production in the leaves inoculated with $P$. syringae pv. tomato DC3000 avrRpt2 (Fig. 2F and I) and DC3000 avrRps4 (Fig. 2G and J).

\section{$A P 2 M$ deficiency reduced DAB gene induction levels.}

Plant defense is associated with transcriptional upregulation of pathogenesis-related $(P R)$ genes such as PRl (At2g14610) and PR2 (At3g57260) (van Loon et al. 2006). We compared the levels of $P R 1$ and $P R 2$ mRNAs in wild-type, ap $2 m$, and $A P 2 M-G F P$ ap $2 m$ plants inoculated with $P$. syringae pv. tomato DC3000 avrRpm1. PR1 and PR2 expression in wildtype plants was induced $3 \mathrm{~h}$ after bacterial inoculation and remained elevated $12 \mathrm{~h}$ after the inoculation (Fig. 3A). Relative to the wild-type expression levels $12 \mathrm{~h}$ after the inoculation of $P$. syringae pv. tomato DC3000 avrRpml, PR1 and $P R 2$ expression levels in ap $2 m$ plants were statistically lower (Fig. 3A). The reduced expression of $P R 1$ and $P R 2$ in ap $2 m$ was rescued by expression of AP2M-GFP, confirming that the transcriptional defect of $P R$ genes is caused by the mutation in AP2M (Fig. 3A). These results indicate that AP2M is involved in induction of $P R-1$ and $P R-2$ during RPM1mediated ETI. Similar reductions in the $P R-1$ and $P R-2$ induced levels were observed in ap $2 \mathrm{~m}$ plants after $P$. syringae pv. tomato DC3000 avrRpt2 inoculation (Fig. 3B). In contrast, the ap $2 m$ plants inoculated with DC3000 avrRps4 showed induced levels of $P R 1$ and $P R 2$ very similar to those of wild-type and $A P 2 M$ GFP ap $2 m$ plants (Fig. 3C).

\section{DISCUSSION}

We have reported here that AP2M is partially required for both RPM1-mediated and RPS2-mediated ETI but not required for RPS4-mediated ETI. AP2M is the $\mu$ subunit of the evolutionarily conserved AP- 2 complex, which plays a central role in CME from the plasma membrane (Di Rubbo et al. 2013; Fan et al. 2013; Kim et al. 2013; Yamaoka et al. 2013). Our results suggest that CME is involved in the signaling cascade for RPM1- and RPS2-mediated immune responses, whereas the RPS4-mediated immune response is independent of CME. Endocytosis in some cases attenuates signaling by removing activated receptors from the plasma membrane (Gagliardi et al. 2008). It is also likely that endosomes act as platforms for active signaling and that endocytosis plays a crucial role in signal propagation and amplification (Gagliardi et al. 2008). CRT1, a member of the GHKL ATPase/kinase superfamily (Dutta and Inouye 2000), physically interacted with both RPM1 and RPS2 (Kang et al. 2010). An Arabidopsis mutant lacking CRT1 displayed compromised resistance to $P$. syringae pv. tomato DC3000 avrRpm1 and DC3000 avrRpt2 (Kang et al. 2010). CRT1 localized to endosomes. This implies that the interactions of CRT1 with RPM1 and RPS2 occur in endosomes (Kang et al. 2010), whereas it has been suggested that 
RPM1 functions on the plasma membrane (Gao et al. 2011). RPM1 and RPS2 have been shown to form complexes on the plasma membrane with plasma membrane-associated proteins (Qi and Katagiri 2009). These reports suggest that RPM1, RPS2, or other receptor complex components can relocate from the plasma membrane to endosomes and mediate immune signal transduction in endosomes. In support of this hypothesis, the potato NB-LRR immune receptor R3a localizes to endosomes for recognition of the Phytophthora infestans effector AVR3a(KI) (Engelhardt et al. 2012). Endosomal localization of these immune receptors might be mediated by the AP-2 complex. Future identification of the cargo proteins of AP-2 will likely unravel the role of AP-2-dependent endocytosis in the plant immune response. However, it should be noted that the effect of the ap $2 m$ mutation on RPM1- and RPS2-mediated ETI and its associated responses was partial. While endosomal localization of one or more immune signaling components may be important to express full-strength RPM1- and RPS2mediated ETI, there appears to also be one or more other mechanisms that mediate signaling downstream of the plasma membrane.

AP2M deficiency compromised ETI against $P$. syringae pv. tomato DC3000 avrRpm1 and DC3000 avrRpt2, which was associated with both weakened hypersensitive cell death and $P R$ gene induction. In contrast, ROS production was intact in the ap $2 m$ mutant during ETI. There are at least three hypotheses to explain these observations. One possible explanation is that the effector recognition functions are normal in the ap $2 m$ mutants but the downstream pathway of ROS production is impaired. Recent studies suggest that endocytosis plays a role in immune responses downstream of ROS production. Cryptogein stimulated CME in a ROS-dependent manner in tobacco BY-2 cells. This event was abolished in a cell line with impaired ROS production (Leborgne-Castel et al. 2008). Another study showed that inhibition of ROS produced in response to flg22 prevented FLS2 endocytosis (Serrano et al. 2007). These observations may suggest that AP2M functions in the pathway between ROS production and CME. A second possible explanation is that the signaling pathway downstream of effector recognition is divided into two separate branches, one affected by the ap $2 \mathrm{~m}$ mutation and the other leading to ROS production. Consistent with this notion, the ROS production was strongly reduced but the HR was only moderately affected in an Arabidopsis rbohD mutant in response to $P$. syringae pv. tomato DC3000 avrRpml (Torres et al. 2002). Similarly, Nbrboh-silenced Nicotiana benthamiana plants exhibited reduced elicitor-mediated ROS production but did not show an obvious change in HR or $P R$ gene expression compared with nonsilenced plants (Zhang et al. 2009). In Arabidopsis, a defect in proteasome function did not affect ROS production but abolished HR associated with ETI (Hatsugai et al. 2009). Thus, ROS production may be mediated by a different signaling pathway than cell death and other responses during ETI. A third possible explanation is based on the observation that the effect of the ap2 $m$ mutation is quantitative. Both RPM1-mediated and RPS2-mediated ETI were only partially abolished in the ap $2 m$ mutant, unlike in the corresponding $R$ gene mutants. A weak signal for ETI must still remain even in the ap $2 m$ mutant. This weak signal might be sufficient to fully induce ROS production as the signal-dose response for ROS production may have a low saturation point. Other ETI-associated responses were, however, affected; they may have higher saturate points in the signal-dose response.

Taken together, our results demonstrate that AP2M is important but not absolutely required for plant immune responses. They also suggest that AP2M is required for full resistance mediated by membrane-localized $\mathrm{R}$ proteins.

\section{MATERIALS AND METHODS}

Plant materials and growth conditions.

Arabidopsis thaliana accession Col-0 was the background of all the plants used in this study. Arabidopsis ap2m (SAIL_165_A02) (Yamaoka et al. 2013), rpml-3 (Grant et al. 1995), rps2 101C (Mindrinos et al. 1994), rpm1-3/rps2 101C (Mackey et al. 2003), rps4 (SALK_057697) (Wirthmueller et al. 2007), and AP2M-GFP ap $2 m$ (Yamaoka et al. 2013) were previously described. Arabidopsis plants were grown in a controlled environment at $22^{\circ} \mathrm{C}$ with a 12 -h photoperiod and $75 \%$ relative humidity.

\section{Bacterial strains and preparation of inocula.}

P. syringae pv. tomato DC3000 was grown overnight in King's B medium supplemented with $50 \mu \mathrm{g}$ of rifampicin per milliliter. $P$. syringae pv. tomato DC3000 strains carrying the empty vector (pVSP61), avrRpm1, avrRpt2, or avrRps4 were grown overnight in King's B medium supplemented with $50 \mu \mathrm{g}$ of rifampicin and $30 \mu \mathrm{g}$ of kanamycin per milliliter. The bacteria were harvested by centrifugation, were washed, and were diluted to the desired density with water.

\section{Bacterial growth assay.}

Bacterial growth assays were performed as described previously (Tsuda et al. 2008). In brief, bacterial suspensions of the indicated density were infiltrated into leaves of 4- to 5-week-old plants using a needleless syringe. CFU per square centimeter of leaf surface area were counted from leaf tissue collected 2 days after infiltration. A mixed-effect model was fit to $\log _{10^{-}}$ transformed bacterial count data, using the lme function from the nlme package in the R environment. The model $S_{\text {gtrf }}=$ $G T_{g t}+R_{r} / F_{f}+\varepsilon_{g t r f}$, where $S=\log _{10}$-transformed bacterial number, $G T=$ plant_genotype:treatment interaction (different treatments represent different bacterial strains), and random factors, $R=$ biological replicate (from independent experiments); $F=$ planting flat nested within each replicate, and $\varepsilon=$ residual. The mean estimates of the genotype:treatment interactions were used as the modeled $\log _{10}$-transformed bacterial counts. Bacterial growth was calculated as the difference in modeled $\log _{10}$-transformed bacterial counts between each mutant genotype and wild-type genotype. Growth values of effector and empty vector strains were compared using two-tailed $t$ tests. For the $t$ tests, the standard errors appropriate for each comparison of differences were calculated using the variance and covariance values obtained from the model fitting. $P$ values obtained from the $t$ tests were adjusted using the Holm-Bonferroni multiple testing correction.

\section{Quantitative reverse transcription-polymerase chain reaction (qRT-PCR) analysis.}

Total RNA was isolated using TRIzol (Invitrogen) according to the manufacturer's protocol. qRT-PCR was performed using a Superscript III Platinum SYBR Green One-Step qRT-PCR kit (Invitrogen) and a Roche LightCycler480 machine. Primers for qRT-PCR are listed in Supplementary Table S1. The relative $\log _{2}$ expression values were obtained by subtracting the cycle threshold $\left(C_{\mathrm{t}}\right)$ values for each gene from the corresponding $C_{\mathrm{t}}$ values of the Actin 2 gene. A mixed-effect model was fit separately to the relative $\log _{2}$ expression data for each gene, using the lme function from the nlme package in the $\mathrm{R}$ environment. The model $\Delta C t_{\text {gtir }}=G T I_{g t i}+R_{r} / T_{t} / I_{i}+\varepsilon_{\text {gtir }}$, where $G T I=$ genotype: treatment:time interaction, and random factors, $R=$ biological replicate (from independent experiments), $T=$ treatment nested within replicate, $I=$ time nested within treatment, and $\varepsilon=$ residual. The mean estimates of the genotype:treatment:time interactions were used as the modeled $\log _{2}$ expression values. Modeled $\log _{2}$ expression values for the mutant genotypes were 
compared with those of the wild-type genotype, using two-tailed $t$ tests. For these $t$ tests, the relevant standard error for each comparison was calculated using the variance and covariance values obtained from the model fitting. For each gene independently, $P$ values obtained from the $t$ tests were adjusted for multiple hypothesis testing using the Holm-Bonferroni correction.

\section{Trypan blue staining.}

Inoculated leaves were boiled for approximately $1 \mathrm{~min}$ in the staining solution ( $1 \mathrm{ml}$ of lactic acid, $1 \mathrm{ml}$ of glycerol, $1 \mathrm{ml}$ of water-saturated phenol, $1 \mathrm{ml}$ of water, and $1 \mathrm{mg}$ of trypan blue) and were incubated overnight at room temperature. Then, samples were decolorized in $2.5 \mathrm{~g}$ of chloral hydrate per milliliter for at least 1 day.

\section{Electrolyte leakage assay.}

Electrolyte leakage from dying and dead cells was measured essentially as described previously (Hatsugai et al. 2009). Four discs with a 7.5-mm diameter were dissected from leaves immediately after bacterial inoculation, were floated in $2 \mathrm{ml}$ of distilled water for $30 \mathrm{~min}$, were transferred into $2 \mathrm{ml}$ of distilled water, and were incubated at $22^{\circ} \mathrm{C}$. The conductance of water was measured with an electrical conductivity meter (B-173; Horiba)

\section{Reactive oxygen species (ROS) detection.}

ROS was visualized in leaf tissues by staining with DAB (Sigma-Aldrich), as described previously (Thordal-Christensen et al. 1997). Leaves were vacuum-infiltrated with DAB solution. The leaf samples were placed in a plastic box with high humidity overnight at room temperature. Leaves were fixed $(60 \%$ [vol/vol] ethanol, $20 \%$ [vol $/ \mathrm{vol}]$ lactic acid, and $20 \%$ [vol $/ \mathrm{vol}]$ glycerol), were decolorized in $2.5 \mathrm{~g}$ chloral hydrate per milliliter, and were visualized with a light microscope. The grayscale images of the DAB-stained leaves were used to quantify the signal intensity using Fiji/ImageJ software ver. 1.47q (Schindelin et al. 2012).

\section{ACKNOWLEDGMENTS}

We are grateful to the Arabidopsis Biological Resource Center at Ohio State University for providing the Arabidopsis T-DNA insertion mutant seeds. This work was supported by a grant from the National Science Foundation, IOS-1121425 to F.K., by Grants-in-Aid for Scientific Research (grant numbers 22000014 to I. Hara-Nishimura and 23570043 to N. Hatsugai) from the Ministry of Education, Culture, Sports, Science and Technology (MEXT) of Japan, by the Sumitomo Foundation (N. Hatsugai) and by the Global Center of Excellence Program "Formation of a Strategic Base for Biodiversity and Evolutionary Research: From Genome to Ecosystem" of MEXT (I. Hara-Nishimura).

\section{LITERATURE CITED}

Axtell, M. J., and Staskawicz, B. J. 2003. Initiation of RPS2-specified disease resistance in Arabidopsis is coupled to the AvrRpt2-directed elimination of RIN4. Cell 112:369-377.

Bar, M., and Avni, A. 2014. Endosomal trafficking and signaling in plant defense responses. Curr. Opin. Plant Biol. 22:86-92.

Barberon, M., Zelazny, E., Robert, S., Conejero, G., Curie, C., Friml, J., and Vert, G. 2011. Monoubiquitin-dependent endocytosis of the ironregulated transporter 1 (IRT1) transporter controls iron uptake in plants. Proc. Natl. Acad. Sci. U.S.A. 108:E450-458.

Chen, F., Gao, M. J., Miao, Y. S., Yuan, Y. X., Wang, M. Y., Li, Q., Mao, B. Z., Jiang, L. W., and He, Z. H. 2010. Plasma membrane localization and potential endocytosis of constitutively expressed XA21 proteins in transgenic rice. Mol. Plant 3:917-926.

Dangl, J. L., and Jones, J. D. 2001. Plant pathogens and integrated defence responses to infection. Nature 411:826-833.

Dhonukshe, P., Aniento, F., Hwang, I., Robinson, D. G., Mravec, J., Stierhof, Y. D., and Friml, J. 2007. Clathrin-mediated constitutive endocytosis of PIN auxin efflux carriers in Arabidopsis. Curr. Biol. 17: 520-527.
Di Rubbo, S., Irani, N. G., Kim, S. Y., Xu, Z. Y., Gadeyne, A., Dejonghe, W., Vanhoutte, I., Persiau, G., Eeckhout, D., Simon, S., Song, K., Kleine-Vehn, J., Friml, J., De Jaeger, G., Van Damme, D., Hwang, I., and Russinova, E. 2013. The clathrin adaptor complex AP-2 mediates endocytosis of brassinosteroid insensitive 1 in Arabidopsis. Plant Cell 25:2986-2997.

Dutta, R., and Inouye, M. 2000. GHKL, an emergent ATPase/kinase superfamily. Trends Biochem. Sci. 25:24-28.

Ebine, K., Fujimoto, M., Okatani, Y., Nishiyama, T., Goh, T., Ito, E., Dainobu, T., Nishitani, A., Uemura, T., Sato, M. H., Thordal-Christensen, H., Tsutsumi, N., Nakano, A., and Ueda, T. 2011. A membrane trafficking pathway regulated by the plant-specific RAB GTPase ARA6. Nat. Cell Biol. 13:853-859.

Engelhardt, S., Boevink, P. C., Armstrong, M. R., Ramos, M. B., Hein, I., and Birch, P. R. 2012. Relocalization of late blight resistance protein R3a to endosomal compartments is associated with effector recognition and required for the immune response. Plant Cell 24:5142-5158.

Fan, L., Hao, H., Xue, Y., Zhang, L., Song, K., Ding, Z., Botella, M. A. Wang, H., and Lin, J. 2013. Dynamic analysis of Arabidopsis AP2 $\sigma$ subunit reveals a key role in clathrin-mediated endocytosis and plant development. Development 140:3826-3837.

Gagliardi, M., Piddini, E., and Vincent, J. P. 2008. Endocytosis: A positive or a negative influence on Wnt signalling? Traffic 9:1-9.

Gao, Z., Chung, E. H., Eitas, T. K., and Dangl, J. L. 2011. Plant intracellular innate immune receptor Resistance to Pseudomonas syringae pv maculicola 1 (RPM1) is activated at, and functions on, the plasma membrane. Proc. Natl. Acad. Sci. U.S.A. 108:7619-7624.

Gassmann, W., Hinsch, M. E., and Staskawicz, B. J. 1999. The Arabidopsis RPS4 bacterial-resistance gene is a member of the TIR-NBS-LRR family of disease-resistance genes. Plant J. 20:265-277.

Geldner, N., and Robatzek, S. 2008. Plant receptors go endosomal: A moving view on signal transduction. Plant Physiol. 147:1565-1574.

Grant, M. R., Godiard, L., Straube, E., Ashfield, T., Lewald, J., Sattler, A., Innes, R. W., and Dangl, J. L. 1995. Structure of the Arabidopsis RPM1 gene enabling dual specificity disease resistance. Science 269:843-846

Hara-Nishimura, I., and Hatsugai, N. 2011. The role of vacuole in plant cell death. Cell Death Differ. 18:1298-1304.

Hatsugai, N., Iwasaki, S., Tamura, K., Kondo, M., Fuji, K., Ogasawara, K., Nishimura, M., and Hara-Nishimura, I. 2009. A novel membrane fusionmediated plant immunity against bacterial pathogens. Genes Dev. 23: 2496-2506.

Irani, N. G., Di Rubbo, S., Mylle, E., Van den Begin, J., Schneider-Pizon, J., Hnilikova, J., Sisa, M., Buyst, D., Vilarrasa-Blasi, J., Szatmari, A. M., Van Damme, D., Mishev, K., Codreanu, M. C., Kohout, L., Strnad, M. Cano-Delgado, A. I., Friml, J., Madder, A., and Russinova, E. 2012. Fluorescent castasterone reveals BRI1 signaling from the plasma membrane. Nat. Chem. Biol. 8:583-589.

Jones, J. D., and Dangl, J. L. 2006. The plant immune system. Nature 444: 323-329.

Jones, D. A., Thomas, C. M., Hammond-Kosack, K. E., Balint-Kurti, P. J., and Jones, J. D. 1994. Isolation of the tomato Cf-9 gene for resistance to Cladosporium fulvum by transposon tagging. Science 266:789-793.

Kang, H. G., Oh, C. S., Sato, M., Katagiri, F., Glazebrook, J., Takahashi, H., Kachroo, P., Martin, G. B., and Klessig, D. F. 2010. Endosome-associated CRT1 functions early in resistance gene-mediated defense signaling in Arabidopsis and tobacco. Plant Cell 22:918-936.

Kankanala, P., Czymmek, K., and Valent, B. 2007. Roles for rice membrane dynamics and plasmodesmata during biotrophic invasion by the blast fungus. Plant Cell 19:706-724.

Kawchuk, L. M., Hachey, J., Lynch, D. R., Kulcsar, F., van Rooijen, G., Waterer, D. R., Robertson, A., Kokko, E., Byers, R., Howard, R. J., Fischer, R., and Prufer, D. 2001. Tomato Ve disease resistance genes encode cell surface-like receptors. Proc. Natl. Acad. Sci. U.S.A. 98: 6511-6515

Kim, S. Y., Xu, Z. Y., Song, K., Kim, D. H., Kang, H., Reichardt, I., Sohn, E. J., Friml, J., Juergens, G., and Hwang, I. 2013. Adaptor protein complex 2-mediated endocytosis is crucial for male reproductive organ development in Arabidopsis. Plant Cell 25:2970-2985.

Leborgne-Castel, N., Lherminier, J., Der, C., Fromentin, J., Houot, V., and Simon-Plas, F. 2008. The plant defense elicitor cryptogein stimulates clathrin-mediated endocytosis correlated with reactive oxygen species production in bright yellow-2 tobacco cells. Plant Physiol. 146:1255-1266.

Leborgne-Castel, N., Adam, T., and Bouhidel, K. 2010. Endocytosis in plant-microbe interactions. Protoplasma 247:177-193.

Mackey, D., Belkhadir, Y., Alonso, J. M., Ecker, J. R., and Dangl, J. L. 2003. Arabidopsis RIN4 is a target of the type III virulence effector AvrRpt2 and modulates RPS2-mediated resistance. Cell 112:379389. 
Mindrinos, M., Katagiri, F., Yu, G. L., and Ausubel, F. M. 1994. The A thaliana disease resistance gene RPS 2 encodes a protein containing a nucleotide-binding site and leucine-rich repeats. Cell 78:1089-1099.

Ohno, H., Stewart, J., Fournier, M. C., Bosshart, H., Rhee, I., Miyatake, S., Saito, T., Gallusser, A., Kirchhausen, T., and Bonifacino, J. S. 1995. Interaction of tyrosine-based sorting signals with clathrin-associated proteins. Science 269:1872-1875.

Qi, Y., and Katagiri, F. 2009. Purification of low-abundance Arabidopsis plasma-membrane protein complexes and identification of candidate components. Plant J. 57:932-944.

Ron, M., and Avni, A. 2004. The receptor for the fungal elicitor ethyleneinducing xylanase is a member of a resistance-like gene family in tomato. Plant Cell 16:1604-1615.

Schindelin, J., Arganda-Carreras, I., Frise, E., Kaynig, V., Longair, M., Pietzsch, T., Preibisch, S., Rueden, C., Saalfeld, S., Schmid, B., Tinevez, J. Y., White, D. J., Hartenstein, V., Eliceiri, K., Tomancak, P., and Cardona, A. 2012. Fiji: An open-source platform for biological-image analysis. Nat. Methods 9:676-682.

Serrano, M., Robatzek, S., Torres, M., Kombrink, E., Somssich, I. E., Robinson, M., and Schulze-Lefert, P. 2007. Chemical interference of pathogen-associated molecular pattern-triggered immune responses in Arabidopsis reveals a potential role for fatty-acid synthase type II complex-derived lipid signals. J. Biol. Chem. 282:6803-6811.

Sharfman, M., Bar, M., Ehrlich, M., Schuster, S., Melech-Bonfil, S., Ezer, R., Sessa, G., and Avni, A. 2011. Endosomal signaling of the tomato leucine-rich repeat receptor-like protein LeEix2. Plant J. 68:413-423.

Takano, J., Tanaka, M., Toyoda, A., Miwa, K., Kasai, K., Fuji, K., Onouchi, H., Naito, S., and Fujiwara, T. 2010. Polar localization and degradation of Arabidopsis boron transporters through distinct trafficking pathways. Proc. Natl. Acad. Sci. U.S.A. 107:5220-5225.

Takken, F. L., Schipper, D., Nijkamp, H. J., and Hille, J. 1998. Identification and $D s$-tagged isolation of a new gene at the $C f$ - 4 locus of tomato involved in disease resistance to Cladosporium fulvum race 5. Plant J. 14:401-411.

Tameling, W. I., and Joosten, M. H. 2007. The diverse roles of NB-LRR proteins in plants. Physiol. Mol. Plant Pathol. 71:126-134.
Teh, O. K., and Hofius, D. 2014. Membrane trafficking and autophagy in pathogen-triggered cell death and immunity. J. Exp. Bot. 65:1297-1312.

Thordal-Christensen, H., Zhang, Z., Wei, Y., and Collinge, D. B. 1997 Subcellular localization of $\mathrm{H}_{2} \mathrm{O}_{2}$ in plants: $\mathrm{H}_{2} \mathrm{O}_{2}$ accumulation in papillae and hypersensitive response during the barley-powdery mildew. interaction. Plant J. 11:1187-1194.

Tornero, P., Merritt, P., Sadanandom, A., Shirasu, K., Innes, R. W., and Dangl, J. L. 2002. RAR1 and NDR1 contribute quantitatively to disease resistance in Arabidopsis, and their relative contributions are dependent on the $R$ gene assayed. Plant Cell 14:1005-1015.

Torres, M. A., Dangl, J. L., and Jones, J. D. 2002. Arabidopsis gp91phox homologues AtrbohD and AtrbohF are required for accumulation of reactive oxygen intermediates in the plant defense response. Proc. Natl. Acad. Sci. U.S.A. 99:517-522.

Torres, M. A., Jones, J. D., and Dangl, J. L. 2006. Reactive oxygen species signaling in response to pathogens. Plant Physiol. 141:373-378.

Tsuda, K., and Katagiri, F. 2010. Comparing signaling mechanisms engaged in pattern-triggered and effector-triggered immunity. Curr. Opin. Plant Biol. 13:459-465.

Tsuda, K., Sato, M., Glazebrook, J., Cohen, J. D., and Katagiri, F. 2008. Interplay between MAMP-triggered and SA-mediated defense responses. Plant J. 53:763-775.

van Loon, L. C., Rep, M., and Pieterse, C. M. 2006. Significance of inducible defense-related proteins in infected plants. Annu. Rev. Phytopathol. 44: 135-162.

Wirthmueller, L., Zhang, Y., Jones, J. D., and Parker, J. E. 2007. Nuclear accumulation of the Arabidopsis immune receptor RPS4 is necessary for triggering EDS1-dependent defense. Curr. Biol. 17:2023-2029.

Yamaoka, S., Shimono, Y., Shirakawa, M., Fukao, Y., Kawase, T., Hatsugai, N., Tamura, K., Shimada, T., and Hara-Nishimura, I. 2013. Identification and dynamics of Arabidopsis adaptor protein-2 complex and its involvement in floral organ development. Plant Cell 25:2958-2969.

Zhang, H., Fang, Q., Zhang, Z., Wang, Y., and Zheng, X. 2009. The role of respiratory burst oxidase homologues in elicitor-induced stomatal closure and hypersensitive response in Nicotiana benthamiana. J. Exp. Bot. 60:3109-3122. 